\title{
Penalized-likelihood image reconstruction for digital holography
}

\author{
Saowapak Sotthivirat \\ National Electronics and Computer Development Center, National Science and Technology Development Agency, \\ Ministry of Science and Technology, Klong Luang, Pathumthani 12120, Thailand \\ Jeffrey A. Fessler \\ Department of Electrical Engineering and Computer Science, University of Michigan, Ann Arbor, \\ Michigan 48109-2122
}

Received March 10, 2003; revised manuscript received December 16, 2004; accepted December 17, 2004

\begin{abstract}
Conventional numerical reconstruction for digital holography using a filter applied in the spatial-frequency domain to extract the primary image may yield suboptimal image quality because of the loss in high-frequency components and interference from other undesirable terms of a hologram. We propose a new numerical reconstruction approach using a statistical technique. This approach reconstructs the complex field of the object from the real-valued hologram intensity data. Because holographic image reconstruction is an ill-posed problem, our statistical technique is based on penalized-likelihood estimation. We develop a Poisson statistical model for this problem and derive an optimization transfer algorithm that monotonically decreases the cost function at each iteration. Simulation results show that our statistical technique has the potential to improve image quality in digital holography relative to conventional reconstruction techniques. (c) 2004 Optical Society of America
\end{abstract}

OCIS codes: $090.0090,090.1760,100.0100,100.2000,100.3010,100.3190$.

\section{INTRODUCTION}

Holography is a technique for recording and reconstructing both the amplitude and the phase of a wave field. ${ }^{1}$ Analog conventional holography consists of two optical processes: recording and reconstructing. First, the interference pattern between an object wave front and a reference beam is recorded with photographic material. The recorded pattern is called a hologram. Then, one reconstructs the object's complex wave front by illuminating the recording medium with a wave that is similar to the original reference beam. This process generates the zero-order image and the twin images called the primary (virtual) and conjugate (real) images. In in-line holography invented by Gabor, ${ }^{2}$ the reconstructed holographic image suffers from an overlap of these three images, degrading resolution and contrast. To separate the primary image from other terms, Leith and Upatnieks ${ }^{3}$ invented off-axis holography by introducing the reference beam at an angle with respect to the object beam. With this technique, the zero-order, primary, and conjugate images appear at different locations, so each image can be observed separately.

Because the processes of optical recording on photographic film and optical reconstruction preparation in analog conventional holography are time-consuming and lack real-time imaging ability, digital recording of a hologram on a digital detector (such as a charge-coupleddevice (CCD) camera) and numerical reconstruction of a complex object field on a computer become attractive alternatives and have been useful in many applications. ${ }^{4-8}$ In digital off-axis holography, the most common approach for extracting the primary image in numerical reconstruction is to perform a digital "spatial filter" that selects appropriate spatial frequencies in the Fourier domain of a hologram. ${ }^{9}$ Limitations of that approach include a loss of high-frequency components and interference from other terms in the hologram, which degrade the reconstructed holographic image quality. Phase-shifting or phase modulation ${ }^{7,10,11}$ methods were proposed to suppress the zero-order and conjugate images, but they require at least three holograms to reconstruct one holographic image. The clever approach proposed in Ref. 12 estimates the complex object beam by solving a small system of equations; however, no noise model was considered.

To overcome the drawbacks of existing approaches, in this paper we propose a new numerical holographic reconstruction approach based on a statistical model for the measurements and a physical model of the optical system. Statistical image formation techniques have succeeded in many applications. ${ }^{13-16}$ Statistical image reconstruction for digital holography can be formulated as an inverse problem in which we try to obtain a complex reconstructed holographic image (primary image) from hologram intensity data that are real.

Çetin et al. ${ }^{17}$ proposed a statistical technique for Fourier holography and other coherent imaging applications. Their method was based on a Gaussian noise model and used a least-squares approach. Considering the digital recording process of a hologram, our statistical model follows a Poisson distribution having the mean associated with a squared magnitude of the interference between the object and reference beams. Because of the ill-posed na- 
ture of image reconstruction, our statistical technique uses penalized-likelihood (PL) estimation. ${ }^{18-20}$ This optimization problem is challenging because its negative log-likelihood function contains multiple global minima. Therefore regularization is necessary to improve the problem conditioning and to reduce nonuniqueness. Moreover, we show that using two measured holograms can improve the results when reconstructing a complex holographic image with the same number of pixels as the recording device. (The use of two data sets to help estimate complex quantities has been applied in other optical problems. ${ }^{21,22}$ )

In PL estimation, the unknown parameter vector, which represents the complex object field, is estimated by minimizing a cost function. Since closed-form solutions are unavailable, we need an iterative algorithm to solve the problem. However, for the Poisson model, conventional gradient-based minimization is difficult. To simplify the optimization problem and to ensure monotonic decreases in the cost function at each iteration, our proposed reconstruction approach is based on the use of optimization transfer and convexity techniques by finding a "surrogate function" that lies above the original cost function at each iteration. ${ }^{14,23}$ Instead of minimizing the original cost function, we minimize the surrogate function by using an iterative algorithm, such as the separableparaboloidal-surrogate (SPS) ${ }^{14,23}$ or conjugate gradient algorithm.

In this study, we demonstrate our holographic reconstruction method in the specific context of image-plane holography, ${ }^{24,25}$ which is a new imaging technique for forming a three-dimensional (3-D) image of a "thick," partially transparent object without dissection. This technique has the same optical sectioning property as that in confocal scanning microscopy but requires no $x y$ scanning. ${ }^{24,25}$ The proposed reconstruction method can also be applied to phase retrieval problems, to Fourier holography when the system matrix represents the Fourier transform, and to Fresnel holography when the system matrix represents the Fresnel transform.

This paper is organized as follows. Section 2 describes the measurement model of a digitally recorded hologram. Section 3 reviews conventional numerical reconstruction using a spatial filtering technique applied in the frequency domain and introduces iterative reconstruction techniques. Section 4 proposes a statistical model for digital holography and introduces a new statistical holo- graphic reconstruction technique based on PL estimation. Section 5 applies optimization transfer and convexity techniques to derive the surrogate functions and the iterative algorithm for holographic image reconstruction. Section 6 compares different holographic reconstructed images obtained by using our statistical approach and the conventional reconstruction approach. Finally, conclusions are given in Section 7.

\section{MEASUREMENT MODEL FOR DIGITAL HOLOGRAPHY}

For digital holography, a computer performs numerical reconstruction of the object from a hologram that is recorded by a digital detector. Figure 1 illustrates the recording process in digital holography.

We assume a linear relationship between the object beam $u_{o}$ at the hologram (detector) plane and the object field $f$, as described by the following superposition integral:

$$
u_{o}(\mathbf{r})=\int h\left(\mathbf{r} ; \mathbf{r}^{\prime}\right) f^{\text {true }}\left(\mathbf{r}^{\prime}\right) \mathrm{d} \mathbf{r}^{\prime}
$$

where $h\left(\mathbf{r} ; \mathbf{r}^{\prime}\right)$ denotes the continuous-space point-spread function (PSF) of the optical system, $f^{\text {true }}(\mathbf{r})$ denotes the true object, and $\mathbf{r}$ denotes two-dimensional (2-D) spatial coordinates on the recording plane. For a planar object, $\mathbf{r}^{\prime}$ denotes the 2-D coordinates within the object plane. For a 3-D object, $\mathbf{r}^{\prime}$ denotes the 3-D coordinates within the object volume. For $3-\mathrm{D}$ reconstruction in imageplane holography, one must scan the object (or the focal plane) along the optical axis and record a set of holograms. For simplicity, in this paper we focus on the 2-D case. In practice, the recording medium has finite thickness, but we ignore this effect here for simplicity.

For image-plane holography, the PSF $h$ represents the characteristics of the imaging optics. For Fourier holography, $h$ includes a Fourier transform, and for Fresnel holography, $h$ includes a Fresnel transform (an approximation of the diffraction integral). ${ }^{1}$ In all cases, the information about the object $f$ of interest is embedded in the object beam $u_{o}$.

For analog conventional holography, the interference between the object and reference beams at the recording plane has the following continuous-space intensity ${ }^{1}$ :

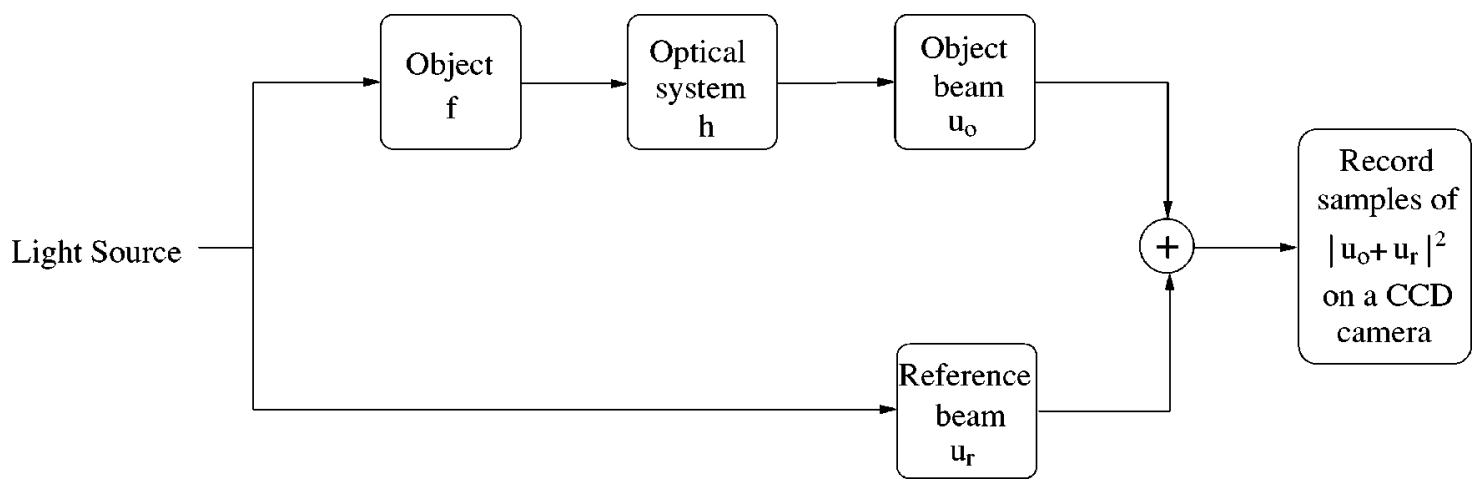

Fig. 1. Diagram of digital holography. 


$$
\begin{aligned}
I(\mathbf{r}) & =\left|u_{o}(\mathbf{r})+u_{\mathrm{ref}}(\mathbf{r})\right|^{2} \\
& =\left|u_{o}(\mathbf{r})\right|^{2}+\left|u_{\mathrm{ref}}(\mathbf{r})\right|^{2}+u_{o}(\mathbf{r}) u_{\mathrm{ref}}^{*}(\mathbf{r})+u_{o}^{*}(\mathbf{r}) u_{\mathrm{ref}}(\mathbf{r}),
\end{aligned}
$$

where $u_{\text {ref }}$ denotes the (known) field of the reference beam and $*$ represents the complex conjugate. The first two terms in Eq. (2) constitute the zero-order image; the third term, which is proportional to $u_{o}$, leads to the formation of the primary image; and the fourth term, which is proportional to $u_{o}^{*}$, leads to the formation of the conjugate image. $^{1}$ For off-axis holography, the reference beam is oriented at some angle resulting in a known spatial carrier frequency denoted by $\boldsymbol{\alpha}$. An example of such a reference beam is a plane wave that is tilted by an angle $\theta$ $=\left(\theta_{x}, \theta_{y}\right)$ in the $x$ and $y$ directions with respect to the optical axis, i.e.,

$$
\begin{aligned}
u_{\mathrm{ref}}(\mathbf{r}) & =U_{\mathrm{ref}} \exp (-i 2 \pi \mathbf{r} \cdot \boldsymbol{\alpha}), \\
\boldsymbol{\alpha} & =\left(\frac{\sin \theta_{x}}{\lambda}, \frac{\sin \theta_{y}}{\lambda}\right),
\end{aligned}
$$

where $U_{\text {ref }}$ denotes the amplitude of the reference wave and $\lambda$ is the wavelength.

Let $\mathbf{Y}=\left(Y_{1}, \ldots, Y_{N}\right)$ denote the (noisy) hologram measurement data recorded by a digital detector, where $N$ denotes the number of measurement elements. We treat the measurement recorded by the $i$ th element of the digital detector as a random variable whose mean is modeled as follows:

$$
E\left(Y_{i}\right)=\left|u_{o}\left(\mathbf{r}_{i}\right)+u_{\mathrm{ref}}\left(\mathbf{r}_{i}\right)\right|^{2}+b_{i}, \quad i=1, \ldots, N,
$$

where $b_{i}$ denotes offsets due to effects such as dark current and $\mathbf{r}_{i}$ denotes the center of the $i$ th detector element. For simplicity, we treat the CCD camera response as a Dirac impulse at the center of each element. One could generalize Eq. (5) to include convolution with a CCD point response function. The reconstruction goal is to estimate the object $f$ from the measurements $\mathbf{Y}$ by using the model (5).

\section{NUMERICAL HOLOGRAPHIC RECONSTRUCTION METHODS}

This section first reviews conventional numerical reconstruction using a filtering method and then introduces iterative reconstruction techniques.

\section{A. Conventional Filtering Approach}

The first step in conventional numerical holographic reconstruction is to apply a "filter" in the frequency domain to extract either the primary or the conjugate image corresponding to one of the last two terms of Eq. (2). This conventional approach assumes that the reference beam is planar, as in Eq. (3). By substituting Eq. (3) into Eq. (2) and taking the Fourier transform, we convert the spatial-frequency spectrum of the recorded interference pattern into an angular spectrum of diffracted waves:

$$
\begin{aligned}
\mathcal{I}(\boldsymbol{\nu})= & \mathcal{I}_{o}(\boldsymbol{\nu})+\left|U_{\text {ref }}\right|^{2} \delta(\boldsymbol{\nu})+U_{\text {ref }} \mathcal{U}_{o}(\boldsymbol{\nu}-\boldsymbol{\alpha}) \\
& +U_{\text {ref }} \mathcal{U}_{o}^{*}(-\boldsymbol{\nu}-\boldsymbol{\alpha}),
\end{aligned}
$$

where $\boldsymbol{\nu}$ denotes 2-D spatial frequencies, $\mathcal{I}_{0}$ denotes the Fourier transform of the intensity of the object beam, $\mathcal{U}_{o}$ denotes the Fourier transform of the field of the object beam, and $\boldsymbol{\alpha}$ was defined in Eq. (4). The zero-order spectrum, consisting of the first two terms of Eq. (6), must be eliminated to avoid having a background bias in the reconstructed image. The two first-order spectra, the last two terms of Eq. (6), lead to the primary and conjugate images, respectively. Figure 2 shows a simulated recorded hologram for a planar reference beam tilted along the $x$ direction, the magnitude of the Fourier transform of the hologram, and the reconstructed image obtained with the conventional technique.

Considering the Fourier transform of the hologram in Fig. 2, one can extract either the primary or the conjugate image by using a mask to select only one of the first-order spectra and then taking the inverse Fourier transform. Because Eq. (6) consists of four terms yet only one term is extracted, one must consider the appropriate dimensions of the reconstructed image. The usual choice is to reconstruct a holographic image that is the same size as that of the CCD array; this requires zero padding in the highspatial-frequency regions surrounding the extracted frequency components. This conventional approach yields a reconstructed image with poorer resolution than the intrinsic recorder resolution owing to the discarded highfrequency components, and it also suffers from degradation by interference from the residual frequency components of other undesirable terms.

For image-plane holography, no further processing of the extracted image is required after computing the inverse Fourier transform. However, for digital Fresnel holography, an additional processing step is required in which the (discretized) Fresnel transform of the image is computed (similarly for Fourier holography). In contrast, the statistical approach described next requires no such postprocessing, since the effects of the Fresnel or the Fourier transform are incorporated into the PSF $h$ in Eq. (1) and hence into the system matrix $\mathbf{A}$ defined in Eq. (9) below.

\section{B. Iterative Reconstruction}

For iterative holographic reconstruction, one must parameterize the continuous-space object in Eq. (1). We approximate the true object $f^{\text {true }}(\mathbf{r})$ by using a linear combination of basis functions as follows ${ }^{26}$ :

$$
f^{\text {true }}(\mathbf{r}) \approx f(\mathbf{r})=\sum_{j=1}^{P} x_{j} \chi_{j}(\mathbf{r})
$$

where $f(\mathbf{r})$ is an approximation of the true object in the continuous space, $x_{j}$ is the unknown complex coefficient of the $j$ th basis function, $P$ is the total number of parameters (e.g., pixels) to be estimated, and $\chi_{j}(\mathbf{r})$ is a basis function. For example, $\chi_{j}(\mathbf{r})$ is usually chosen to be the indicator function over the $j$ th "pixel" in the object plane, and we adopt this choice in our numerical experiments. However, the formulation is general to any choice of basis functions. Combining Eq. (1) and relation (7), we write the object beam $u_{o}\left(\mathbf{r}_{i}\right)$ in the following discrete form: 


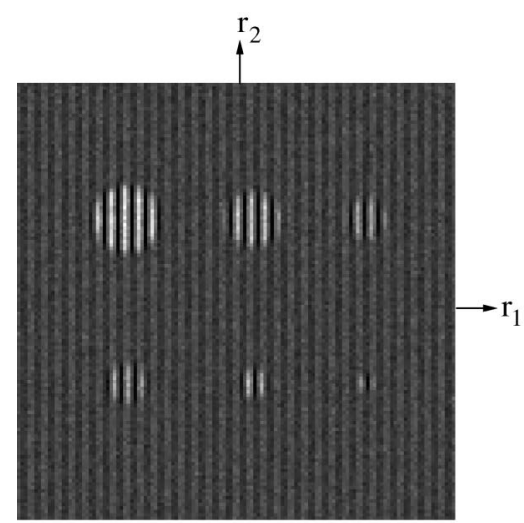

Recorded Hologram

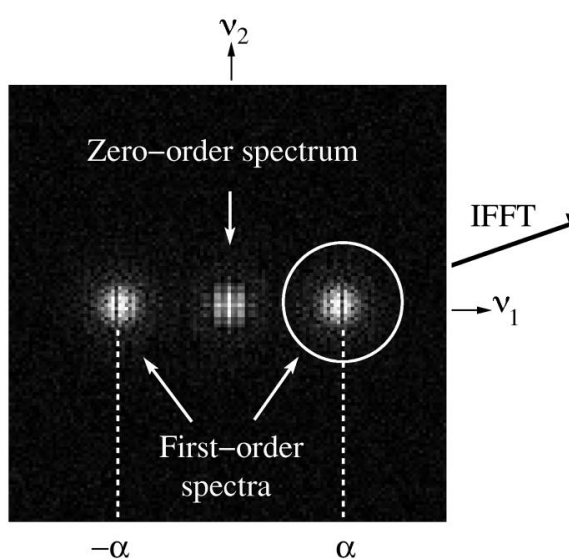

Magnitude of Fourier Transform

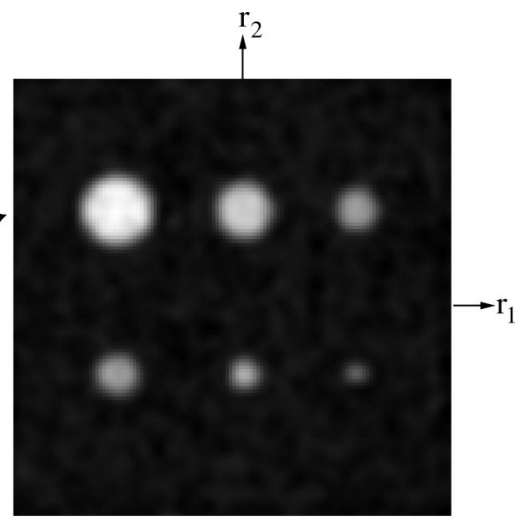

Reconstructed Holographic Image

Fig. 2. Holographic reconstruction using a filtering method. IFFT, inverse fast Fourier transform.

$$
u_{o}\left(\mathbf{r}_{i}\right)=\int h\left(\mathbf{r}_{i} ; \mathbf{r}^{\prime}\right) \sum_{i=1}^{P} x_{j} \chi_{j}\left(\mathbf{r}^{\prime}\right) \mathrm{d} \mathbf{r}^{\prime}=\sum_{j=1}^{P} a_{i j} x_{j}=[\mathbf{A x}]_{i},
$$

where $a_{i j}$ is the $i j$ th element of the system matrix $\mathbf{A}$ and $\mathbf{x}=\left(x_{1}, \ldots, x_{P}\right) . \quad$ Each $a_{i j}$ denotes the contribution of the $j$ th basis function to the object beam $u_{o}\left(\mathbf{r}_{i}\right)$ at the center of the $i$ th recorder element and can be expressed in terms of the system PSF and the basis functions as follows:

$$
a_{i j}=\int h\left(\mathbf{r}_{i} ; \mathbf{r}^{\prime}\right) \chi_{j}\left(\mathbf{r}^{\prime}\right) \mathrm{d} \mathbf{r}^{\prime} .
$$

Combining these expressions with the definition $u_{i} \triangleq u_{\mathrm{ref}}\left(\mathbf{r}_{i}\right)$ leads to the following model for the measurement means:

$$
E\left(Y_{i}\right)=\left|[\mathbf{A x}]_{i}+u_{i}\right|^{2}+b_{i}, \quad i=1, \ldots, N .
$$

We use this model for statistical holographic reconstruction. The goal is to estimate $\mathbf{x}$ from the measured $Y_{i}$ 's, since $\mathbf{x}$ parameterizes the unknown object $f$ of interest. In the special case of space-invariant systems, the system matrix is Toeplitz and $\mathbf{A x}$ corresponds to convolution between the image represented by $\mathbf{x}$ and the discrete-space system PSF.

With the use of Eq. (10), iterative techniques can estimate the complex object field from the measurement data (recorded holograms). Unlike the conventional filtering method, iterative techniques can use all of the information in the model (2) rather than discarding all but one of the four terms. Furthermore, iterative methods need not assume that the reference beam is planar. Therefore iterative methods could work for both in-line and off-axis holography. Since the recorded hologram is real, whereas the unknown object field is complex, if one attempts to estimate a complex holographic image having the same number of pixels as CCD elements, i.e., $P$ $=N$, then the problem will be underdetermined, since each $x_{j}$ in relation (7) is complex valued and so consists of two unknown numbers. Regularization may help reduce this problem. Alternatively, one should choose the number of parameters $P$ (and/or the number of recorded hologram samples $N$ ) such that $N \geqslant P$, so that the problem is not intrinsically underdetermined.
Digital hologram measurements are degraded by noise, so before describing an iterative algorithm for holographic reconstruction in Section 5, we first formulate a statistical model for the noise.

\section{STATISTICAL MODEL}

Statistical techniques for inverse problems require a model for the measurement statistics. In digital holography, the two major noise sources are light quanta statistics characterized by a Poisson distribution and electronic readout noise characterized by a Gaussian distribution. Since Poisson-distributed photon noise is inherent in optical imaging, whereas readout noise depends on detector design, we focus on the Poisson component. ${ }^{27}$ (Readout noise variance can be included in the $b_{i}$ term below if needed. ${ }^{27}$ ) In particular, we model the noisy hologram measurements as having independent Poisson distributions with means described in Eq. (10):

$$
Y_{i} \sim \operatorname{Poisson}\left(\left|[\mathbf{A x}]_{i}+u_{i}\right|^{2}+b_{i}\right), \quad i=1, \ldots, N .
$$

All terms in this expression are assumed known except for the unknown image vector $\mathbf{x}$. Because $\mathbf{x}$ is complex, the problem will be underdetermined if the size of the data vector $\mathbf{Y}$ is less than twice the number of elements of $\mathbf{x}$, i.e., if $N<2 P$.

Since the system matrix $\mathbf{A}$ is usually ill-conditioned in imaging problems, we use PL estimation to reconstruct the holographic image by finding the minimizer $\hat{\mathbf{x}}$ of a cost function of the following form:

$$
\Phi(\mathbf{x})=L(\mathbf{x})+V(\mathbf{x}),
$$

where $L$ denotes the negative log-likelihood function corresponding to the statistical model and $V$ denotes a roughness penalty function. Our focus is image-plane holography, which uses incoherent illumination. For imaging with highly coherent illumination, one may need to consider the effects of speckle when designing the roughness penalty. ${ }^{28,29}$

Ignoring irrelevant constants independent of $\mathbf{x}$, the negative log-likelihood function corresponding to the statistical model (11) is given by 


$$
L(\mathbf{x})=\sum_{i=1}^{N} h_{i}\left([\mathbf{A} \mathbf{x}]_{i}\right),
$$

where $h_{i}$ is a real function of a complex scalar argument defined as follows:

$$
h_{i}(l) \triangleq-y_{i} \log \left(\left|l+u_{i}\right|^{2}+b_{i}\right)+\left(\left|l+u_{i}\right|^{2}+b_{i}\right),
$$

where $l$ denotes a general complex argument. It will be convenient to also write $h_{i}$ as an explicit function of the real and imaginary components of its argument, i.e., $h_{i}(l)=h_{i}\left(l^{R}, l^{I}\right)$, where $l=l^{R}+\iota l^{I}$, and where the superscripts $R$ and $I$ denote the real and imaginary parts, respectively. Each $y_{i}$ denotes a (real-valued) realization of the Poisson random variable $Y_{i}$.

We consider penalty functions that discourage differences between neighboring object pixels having the following form ${ }^{23}$ :

$$
V(\mathbf{x})=\beta \sum_{i=1}^{r} \psi\left([\mathbf{C} \mathbf{x}]_{i}\right)
$$

where $\psi$ is a potential function that determines the behavior of the penalty function, $\mathbf{C}$ is a penalty matrix that defines adjacent neighboring pairs of pixels, $\beta$ is a regularization parameter that controls the degree of smoothness in the reconstructed image, and $r$ is the number of pairs of neighboring object pixels. Examples of potential functions include the quadratic potential function $\psi(t)$ $=t^{2} / 2$ and nonquadratic potential functions, such $\mathrm{as}^{30}$

$$
\psi(t)=\delta^{2}[|t / \delta|-\log (1+|t / \delta|)],
$$

where $\delta$ is a parameter than controls the degree of edge preservation. ${ }^{30-32}$ The smaller the $\delta$, the stronger the degree of edge preservation. For simplicity, the penalty matrix that we used in this paper consists of horizontal and vertical adjacent neighbors, which is called a firstorder neighborhood. An example of the matrix $\mathbf{C}$ for a $2 \times 2$ image is

$$
\mathbf{C x}=\left[\begin{array}{cccc}
-1 & 1 & 0 & 0 \\
0 & 0 & -1 & 1 \\
-1 & 0 & 1 & 0 \\
0 & -1 & 0 & 1
\end{array}\right]\left(\begin{array}{l}
x_{1} \\
x_{2} \\
x_{3} \\
x_{4}
\end{array}\right)=\left(\begin{array}{l}
x_{2}-x_{1} \\
x_{4}-x_{3} \\
x_{3}-x_{1} \\
x_{4}-x_{2}
\end{array}\right)
$$

Our goal is to estimate $\mathbf{x}$ by finding the minimizer of the cost function:

$$
\hat{\mathbf{x}} \triangleq \underset{\mathbf{x}}{\arg \min } \Phi(\mathbf{x}) .
$$

Since closed-form solutions for the minimizer are unavailable, iterative algorithms are needed.

\section{ALGORITHM}

In this section, we approach the minimization problem by using optimization transfer and convexity techniques. These lead to an iterative algorithm that monotonically decreases the cost function.

\section{A. Optimization Transfer}

Directly minimizing the cost function in Eq. (12) is difficult for nonquadratic $h_{i}$ 's. To simplify the optimization problem and to ensure monotonic decreases in the cost function at each iteration, one can apply an optimization transfer approach by finding a "surrogate" function $\phi$ that lies above the cost function. ${ }^{14,23,31,33}$ At each iteration, we obtain the next estimate by finding the minimizer of the surrogate function,

$$
\mathbf{x}^{n+1} \triangleq \underset{\mathbf{x}}{\arg \min } \phi\left(\mathbf{x} ; \mathbf{x}^{n}\right),
$$

where $\mathbf{x}^{n}$ denotes the estimate at the $n$th iteration.

Choosing a surrogate function $\phi$ that satisfies the following monotonicity condition ensures that the iterates $\mathbf{x}^{n}$ will monotonically decrease the cost function $\Phi^{14,31,33}$ :

$$
\Phi\left(\mathbf{x}^{n}\right)-\Phi(\mathbf{x}) \geqslant \phi\left(\mathbf{x}^{n} ; \mathbf{x}^{n}\right)-\phi\left(\mathbf{x} ; \mathbf{x}^{n}\right) \quad \forall \mathbf{x} \geqslant \mathbf{0} .
$$

Instead of using the condition above, we choose a surrogate function $\phi\left(\mathbf{x} ; \mathbf{x}^{n}\right)$ that satisfies the following sufficient conditions:

$$
\begin{aligned}
& \phi\left(\mathbf{x}^{n} ; \mathbf{x}^{n}\right)=\Phi\left(\mathbf{x}^{n}\right), \\
& \phi\left(\mathbf{x} ; \mathbf{x}^{n}\right) \geqslant \Phi(\mathbf{x}) \quad \forall \mathbf{x} \in \mathbb{C}^{P}, \\
& \left.\frac{\partial}{\partial x_{j}} \phi\left(\mathbf{x} ; \mathbf{x}^{n}\right)\right|_{\mathbf{x}=\mathbf{x}^{n}}=\left.\frac{\partial}{\partial x_{j}} \Phi(\mathbf{x})\right|_{\mathbf{x}=\mathbf{x}^{n}} \quad \forall j,
\end{aligned}
$$

where $\mathrm{C}^{P}$ defines a $P$-dimensional complex space.

Subsection 5.B presents the surrogate functions for the cost function given in Eq. (12).

\section{B. Paraboloidal-Surrogate Functions}

We first focus on the likelihood part. Since quadratic choices for the surrogate $\phi$ are particularly easy to minimize, our goal now is to find a parabola that lies above the negative log-likelihood function. Figure 3 illustrates the

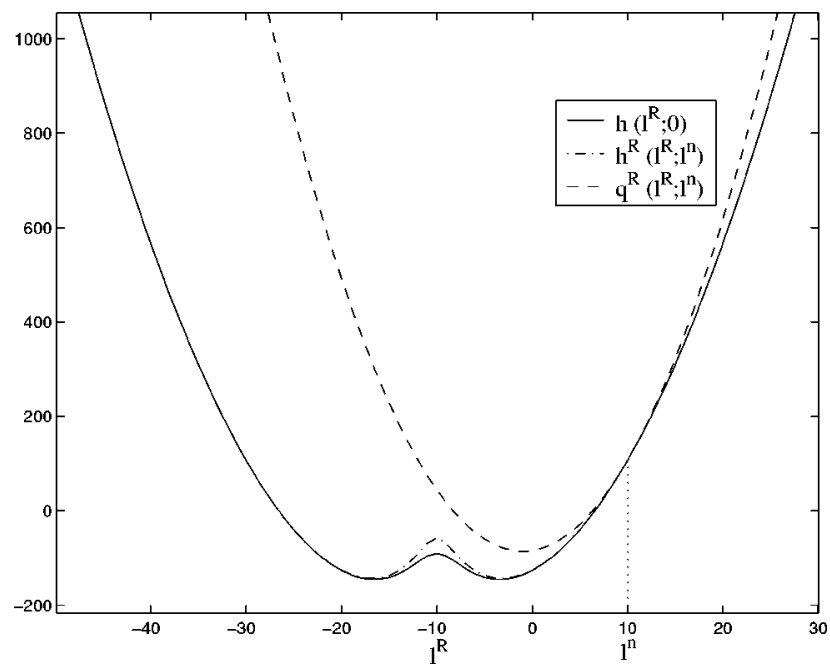

Fig. 3. Illustration of the marginal cost function $h_{i}\left(l^{R}, 0\right)$ and surrogate functions as a function of $l^{R}$. The solid curve is the original marginal cost function. The two other curves lying above the cost function are the surrogate functions. The function denoted by the dashed curve is called the paraboloidalsurrogate function, which has the same first derivative and the same point as those of the original cost function at $l=l^{n}$. 
one-dimensional plot of the marginal cost function $h_{i}\left(l^{R}, 0\right)$. In this plot, the marginal cost function has two optimal minima. However, a 2-D plot of $h_{i}\left(l^{R}, l^{I}\right)$ consists of multiple solutions that lie on a circle in the complex plane. Therefore it is a challenging problem to find the correct optimal solution.

Since $l$ and $u_{i}$ are complex, we adapt De Pierro's multiplicative $\operatorname{trick}^{34}$ to rewrite $h_{i}(l)$ in Eq. (14) as follows:

$$
\begin{aligned}
h_{i}\left(l^{R}, l^{I}\right)= & -y_{i} \log \left\{\alpha_{i}^{R, n}\left[\frac{\left(l^{R}+u_{i}^{R}\right)^{2}+b_{i} / 2}{\alpha_{i}^{R, n}}\right]\right. \\
& \left.+\alpha_{i}^{I, n}\left[\frac{\left(l^{I}+u_{i}^{I}\right)^{2}+b_{i} / 2}{\alpha_{i}^{I, n}}\right]\right\} \\
& +\left[\left(l^{R}+u_{i}^{R}\right)^{2}+b_{i} / 2\right] \\
& +\left[\left(l^{I}+u_{i}^{I}\right)^{2}+b_{i} / 2\right],
\end{aligned}
$$

where we define

$$
\begin{aligned}
\alpha_{i}^{R, n} & =\frac{\left(l^{R, n}+u_{i}^{R}\right)^{2}+b_{i} / 2}{k_{i}^{n}}, \\
\alpha_{i}^{I, n} & =\frac{\left(l^{I, n}+u_{i}^{I}\right)^{2}+b_{i} / 2}{k_{i}^{n}}, \\
k_{i}^{n} & =\left|l_{i}^{n}+u_{i}\right|^{2}+b_{i}, \quad l_{i}^{n}=\left[\mathbf{A x}^{n}\right]_{i} .
\end{aligned}
$$

Combining the fact that $\alpha_{i}^{R, n}+\alpha_{i}^{I, n}=1$ with the convexity of the negative log function in Eq. (20) leads to our first surrogate function, in which we separate the real and imaginary parts,

$$
h_{i}(l)=h_{i}\left(l^{R}, l^{I}\right) \leqslant h_{i}^{R, n}\left(l^{R}\right)+h_{i}^{I, n}\left(l^{I}\right),
$$

where

$$
\begin{aligned}
h_{i}^{R, n}\left(l^{R}\right) \triangleq & -y_{i} \alpha_{i}^{R, n} \log \left[\frac{\left(l^{R}+u_{i}^{R}\right)^{2}+b_{i} / 2}{\alpha_{i}^{R, n}}\right] \\
& +\left(l^{R}+u_{i}^{R}\right)^{2}+b_{i} / 2, \\
h_{i}^{I, n}\left(l^{I}\right) \triangleq & -y_{i} \alpha_{i}^{I, n} \log \left[\frac{\left(l^{I}+u_{i}^{I}\right)^{2}+b_{i} / 2}{\alpha_{i}^{I, n}}\right] \\
& +\left(l^{I}+u_{i}^{I}\right)^{2}+b_{i} / 2 .
\end{aligned}
$$

want to find parabolic surrogates that lie above these curves. A parabolic-surrogate function for the real part has the following form:

$$
\begin{aligned}
q_{i}^{R, n}\left(l^{R}\right)= & h_{i}^{R, n}\left(l_{i}^{R, n}\right)+\dot{h}_{i}^{R, n}\left(l_{i}^{R, n}\right)\left(l^{R}-l_{i}^{R, n}\right) \\
& +\frac{1}{2} c_{i}^{R, n}\left(l^{R}-l_{i}^{R, n}\right)^{2},
\end{aligned}
$$

where $\dot{h}_{i}^{R, n}$ is the first derivative of $h_{i}^{R, n}$ and $c_{i}^{R, n}$ is the curvature of the parabola $q_{i}^{R, n}$. This parabola has the same value as that of $h_{i}^{R, n}$ at the current estimate $l^{R}$ $=l_{i}^{R, n}$ and the same first derivatives at that point. A parabolic-surrogate function for the imaginary part is denoted by $q_{i}^{I, n}\left(l^{I}\right)$ and has an analogous form.

According to the sufficient conditions in expressions (19), we must choose the parabola $q_{i}^{R, n}$ to satisfy the following conditions:

$$
\begin{aligned}
& q_{i}^{R, n}\left(l_{i}^{R, n}\right)=h_{i}^{R, n}\left(l_{i}^{R, n}\right), \\
& q_{i}^{R, n}\left(l^{R}\right) \geqslant h_{i}^{R, n}\left(l^{R, n}\right) \quad \forall l^{R}, \\
& \dot{q}_{i}^{R, n}\left(l_{i}^{R, n}\right)=\dot{h}_{i}^{R, n}\left(l_{i}^{R, n}\right) .
\end{aligned}
$$

Similar conditions apply to $q_{i}^{I, n}$. The first and third conditions are satisfied by the construction of $q_{i}^{R, n}$ and $q_{i}^{I, n}$. The only remaining problem is to find curvatures $c_{i}^{R, n}$ and $c_{i}^{I, n}$ that satisfy the second condition. For the fastest convergence rate, ${ }^{14}$ ideally we would choose the smallest curvature for which the second condition is satisfied. However, a closed-form solution for this optimal choice has proven elusive. Instead, we have chosen the curvatures by using the general expression

$$
c_{i}^{R, n}=\max _{l^{R} \in \mathbb{R}} \frac{\dot{h}_{i}^{R, n}\left(l^{R}\right)-\dot{h}_{i}^{R, n}\left(l_{i}^{R, n}\right)}{l^{R}-l_{i}^{R, n}},
$$

and likewise for $c_{i}^{I, n}$. Although this curvature is not optimal, Appendix A shows that this choice leads to a parabolic surrogate that is guaranteed to lie above the cost function. For the specific model in relation (11), the curvatures in Eq. (25) have the following closed-form solution:

$$
c_{i}^{R, n}=\frac{2 y_{i}\left[b_{i}^{2}+2 b_{i}\left(l_{i}^{R, n}+u_{i}^{R}\right)^{2}\right]^{1 / 2}\left(l_{i}^{R, n}+u_{i}^{R}\right)^{2}}{k_{i}^{n}\left(b_{i}^{2}+b_{i}\left\{2\left(l_{i}^{R, n}+u_{i}^{R}\right)^{2}+\left[b_{i}^{2}+2 b_{i}\left(l_{i}^{R, n}+u_{i}^{R}\right)^{2}\right]^{1 / 2}\right\}\right)}+2 .
$$

In one dimension, the surrogates $h_{i}^{R, n}$ and $h_{i}^{I, n}$ each have two minima (see Fig. 3 ) and are symmetric about the line $l=-u_{i}$. To facilitate the minimization in Eq. (17), we
Appendix B derives this expression. Computation time per iteration could be reduced by using precomputed curvatures. $^{35}$ 
The following inequalities summarize the construction of the surrogate functions:

$$
\begin{aligned}
L(\mathbf{x}) & =\sum_{i=1}^{N} h_{i}\left([\mathbf{A} \mathbf{x}]_{i}\right) \\
& \leqslant \sum_{i=1}^{N} h_{i}^{R, n}\left([\mathbf{A} \mathbf{x}]_{i}^{R}\right)+h_{i}^{I, n}\left([\mathbf{A} \mathbf{x}]_{i}^{I}\right) \\
& \leqslant \sum_{i=1}^{N} q_{i}^{R, n}\left([\mathbf{A} \mathbf{x}]_{i}^{R}\right)+q_{i}^{I, n}\left([\mathbf{A} \mathbf{x}]_{i}^{I}\right) \\
& \triangleq Q\left(\mathbf{x} ; \mathbf{x}^{n}\right) .
\end{aligned}
$$

Since the likelihood surrogate function $Q$ is quadratic, many algorithms could find its minimizer. Minimizing $Q$ is simpler than minimizing the original cost function $\Phi$. In this paper, we apply the separable-paraboloidalsurrogate (SPS) algorithm ${ }^{14,23}$ for this problem. The conjugate gradient method could also be applied easily because nonnegativity constraints are not required.

\section{Separable-Paraboloidal-Surrogate Algorithm}

In this subsection, we derive the SPS algorithm for Eq. (27). To apply the SPS approach, we separate pixels by using the additive convexity technique developed by De Pierro, ${ }^{34}$ permitting simultaneous updates. Using the convexity of $q_{i}^{R, n}$, we have

$$
\begin{aligned}
q_{i}^{R, n}\left([\mathbf{A} \mathbf{x}]_{i}^{R}\right) & =q_{i}^{R, n}\left(\sum_{j=1}^{P} p_{i j}\left(\frac{\left[a_{i j}\left(x_{j}-x_{j}^{n}\right)\right]^{R}}{p_{i j}}+l_{i}^{R, n}\right\}\right) \\
& \leqslant \sum_{j=1}^{P} p_{i j} q_{i}^{R, n}\left(\frac{\left[a_{i j}\left(x_{j}-x_{j}^{n}\right)\right]^{R}}{p_{i j}}+l_{i}^{R, n}\right),
\end{aligned}
$$

where we choose $p_{i j}$ 's that satisfy $p_{i j} \geqslant 0$ and $\sum_{j=1}^{P} p_{i j}$ $=1$. As in previous work, ${ }^{23}$ we use $p_{i j}$ $=\left|a_{i j}\right| / \Sigma_{j^{\prime}=1}^{P}\left|a_{i j^{\prime}}\right|$ in our simulation study. An analogous inequality applies to $q_{i}^{I, n}$. Combining the real and imaginary components leads to the following surrogate function:

$$
\begin{aligned}
Q\left(\mathbf{x} ; \mathbf{x}^{n}\right) & \leqslant \sum_{j=1}^{P} Q_{j}^{n}\left(x_{j}\right) \\
Q_{j}^{n}\left(x_{j}\right) & \triangleq Q_{j}^{R, n}\left(x_{j}\right)+Q_{j}^{I, n}\left(x_{j}\right), \\
Q_{j}^{R, n}\left(x_{j}\right) & \triangleq \sum_{i=1}^{N} p_{i j} q_{i}^{R, n}\left(\frac{\left[a_{i j}\left(x_{j}-x_{j}^{n}\right)\right]^{R}}{p_{i j}}+l_{i}^{R, n}\right),
\end{aligned}
$$

and we define $Q_{j}^{I, n}$ similarly.

To obtain the update at each iteration, we minimize $Q_{j}^{n}\left(x_{j}\right)$. When no penalty is used, we obtain the maximum-likelihood estimate, as is derived in Subsection 5.D.

\section{Maximum-Likelihood Estimation}

Since $Q_{j}^{n}\left(x_{j}\right)=Q_{j}^{n}\left(x_{j}^{R}+\iota x_{j}^{I}\right)$ is a quadratic function of two real variables $x_{j}^{R}$ and $x_{j}^{I}$, we minimize $Q_{j}^{n}$ by using one step of Newton's method, which involves a $2 \times 2$ matrix-vector multiplication for each pixel as follows:

$$
\begin{aligned}
x_{j}^{n+1} & \triangleq \arg \min _{x_{j}} Q_{j}^{n}\left(x_{j}\right) \\
& =x_{j}^{n}-\mathbf{H}_{j}^{-1} \nabla Q_{j}^{n}\left(x_{j}^{n}\right), \quad j=1, \ldots, P,
\end{aligned}
$$

where the gradient of $Q_{j}^{n}$ is defined by

$$
\begin{aligned}
\nabla Q_{j}^{n}\left(x_{j}^{n}\right) \triangleq & \left.\left(\begin{array}{c}
\frac{\partial}{\partial x_{j}^{R}} Q_{j}^{n}\left(x_{j}\right) \\
\frac{\partial}{\partial x_{j}^{I}} Q_{j}^{n}\left(x_{j}\right)
\end{array}\right)\right|_{x_{j}=x_{j}^{n}} \\
& =\left(\begin{array}{c}
\sum_{i=1}^{N} a_{i j}^{R} \dot{h}_{i}^{R, n}\left(l_{i}^{R, n}\right)+a_{i j}^{I} \dot{h}_{i}^{I, n}\left(l_{i}^{I, n}\right) \\
\sum_{i=1}^{N}-a_{i j}^{I} \dot{h}_{i}^{R, n}\left(l_{i}^{R, n}\right)+a_{i j}^{R} \dot{h}_{i}^{I, n}\left(l_{i}^{I, n}\right)
\end{array}\right) \\
& =\left.\left(\begin{array}{c}
\frac{\partial}{\partial x_{j}^{R}} L(\mathbf{x}) \\
\frac{\partial}{\partial x_{j}^{I}} L(\mathbf{x})
\end{array}\right)\right|_{\mathbf{x}=\mathbf{x}^{n}} \triangleq\left(\begin{array}{c}
\dot{L}_{j}^{R, n} \\
\dot{L}_{j}^{I, n}
\end{array}\right)
\end{aligned}
$$

and the $j$ th $2 \times 2$ Hessian matrix $\mathbf{H}_{j}$ is

$$
\begin{aligned}
\mathbf{H}_{j} \triangleq\left[\begin{array}{ll}
d_{j}^{R R} & d_{j}^{R I} \\
d_{j}^{I R} & d_{j}^{I I}
\end{array}\right] & =\left.\left[\begin{array}{cc}
\frac{\partial^{2}}{\partial\left(x_{j}^{R}\right)^{2}} Q_{j}^{n}\left(x_{j}\right) & \frac{\partial^{2}}{\partial x_{j}^{R} \partial x_{j}^{I}} Q_{j}^{n}\left(x_{j}\right) \\
\frac{\partial^{2}}{\partial x_{j}^{I} \partial x_{j}^{R}} Q_{j}^{n}\left(x_{j}\right) & \frac{\partial^{2}}{\partial\left(x_{j}^{I}\right)^{2}} Q_{j}^{n}\left(x_{j}\right)
\end{array}\right]\right|_{x_{j}=x_{j}^{n}} \\
& =\left[\begin{array}{cc}
\sum_{i=1}^{N} \frac{1}{p_{i j}}\left[\left(a_{i j}^{R}\right)^{2} c_{i}^{R, n}+\left(a_{i j}^{I}\right)^{2} c_{i}^{I, n}\right] & \sum_{i=1}^{N} \frac{a_{i j}^{R} a_{i j}^{I}}{p_{i j}}\left(-c_{i}^{R, n}+c_{i}^{I, n}\right) \\
\sum_{i=1}^{N} \frac{a_{i j}^{R} a_{i j}^{I}}{p_{i j}}\left(-c_{i}^{R, n}+c_{i}^{I, n}\right) & \sum_{i=1}^{N} \frac{1}{p_{i j}}\left[\left(a_{i j}^{I}\right)^{2} c_{i}^{R, n}+\left(a_{i j}^{R}\right)^{2} c_{i}^{I, n}\right]
\end{array}\right] .
\end{aligned}
$$


After matrix multiplication, the explicit form for the SPS algorithm becomes simply

$$
\left(\begin{array}{c}
x_{j}^{R, n+1} \\
x_{j}^{I, n+1}
\end{array}\right)=\left(\begin{array}{c}
x_{j}^{R, n}-\frac{1}{\operatorname{det} \mathbf{H}_{j}}\left(d_{j}^{I I} \dot{L}_{j}^{R, n}-d_{j}^{R I} \dot{L}_{j}^{I, n}\right) \\
x_{j}^{I, n}-\frac{1}{\operatorname{det} \mathbf{H}_{j}}\left(-d_{j}^{R I} \dot{L}_{j}^{R, n}+d_{j}^{R R} \dot{L}_{j}^{I, n}\right)
\end{array}\right),
$$

where the determinant of the Hessian matrix $\mathbf{H}_{j}$ is

$$
\operatorname{det} \mathbf{H}_{j}=d_{j}^{R R} d_{j}^{I I}-\left(d_{j}^{R I}\right)^{2} .
$$

The surrogate functions derived in this subsection do not include the penalty function. Without regularization, a noisy image might be obtained after several iterations. Therefore, in Subsection 5.E, we derive the surrogate function for the penalty term in PL estimation. The derivation extends our previous work ${ }^{14,23}$ to the case of complex images.

\section{E. Penalty Surrogate Function and Penalized-Likelihood Estimation}

Lacking any prior information that would relate the real and imaginary parts of the unknown image $\mathbf{x}$, we employ separate penalty functions for the two parts. Since we separately penalize the real and imaginary parts, using different regularization parameters for the real and imaginary parts provides more flexibility than having only one regularization parameter for both. Thus we use a penalty function of the following form:

$$
V(\mathbf{x})=\beta^{R} \sum_{i=1}^{r} \psi\left(\left[\mathbf{C}^{R} \mathbf{x}^{R}\right]_{i}\right)+\beta^{I} \sum_{i=1}^{r} \psi\left(\left[\mathbf{C}^{I} \mathbf{x}^{I}\right]_{i}\right),
$$

where $\mathbf{C}^{R}$ and $\mathbf{C}^{I}$ are penalty matrices for the real and imaginary parts of the estimates and $\beta^{R}$ and $\beta^{I}$ are the corresponding regularization parameters. To preserve edges, we used the nonquadratic potential function $\psi$ in Eq. (16) in our simulations.

Similar to our approach with the nonquadratic likelihood function, we derive the following surrogate functions:

$$
V(\mathbf{x}) \leqslant V^{\prime}\left(\mathbf{x} ; \mathbf{x}^{n}\right) \leqslant \sum_{j=1}^{P} S_{j}^{n}\left(x_{j}\right)
$$

where $V^{\prime}$ is called the paraboloidal-surrogate function for the penalty function and $S_{j}^{n}$ is called the SPS function for the penalty function. The first inequality is derived by forming a parabola that lies above the original penalty function, and the second inequality is derived by using the convexity of $\psi$. If a quadratic potential function is used instead, then the first parabola step is unnecessary. The paraboloidal-surrogate function $V^{\prime}\left(\mathbf{x} ; \mathbf{x}^{n}\right)$ has the following form:

$$
\begin{aligned}
V^{\prime}\left(\mathbf{x} ; \mathbf{x}^{n}\right)= & \beta^{R} \sum_{i=1}^{r} \varphi\left(\left[\mathbf{C}^{R} \mathbf{x}^{R}\right]_{i} ;\left[\mathbf{C}^{R} \mathbf{x}^{R, n}\right]_{i}\right) \\
& +\beta^{I} \sum_{i=1}^{r} \varphi\left(\left[\mathbf{C}^{I} \mathbf{x}^{I}\right]_{i} ;\left[\mathbf{C}^{I} \mathbf{x}^{I, n}\right]_{i}\right),
\end{aligned}
$$

where the function $\varphi(t ; s)$ is a parabola in $t$ for fixed $s$, where $t$ and $s$ denote real scalar arguments and $\varphi$ is defined by

$$
\varphi(t ; s)=\psi(s)+\dot{\psi}(s)(t-s)+\frac{1}{2} \omega(s)(t-s)^{2},
$$

with the curvature $\omega$ of the parabola surrogate ${ }^{32}$ given by

$$
\omega(s)=\frac{\dot{\psi}(s)}{s} .
$$

Since the paraboloidal-surrogate function $V^{\prime}$ is convex, we apply the additive convexity technique developed by De Pierro ${ }^{34}$ to obtain the SPS function that lies above $V^{\prime}$ as follows:

$$
\begin{aligned}
& S_{j}^{R, n}\left(x_{j}^{R}\right) \\
& \triangleq \beta^{R} \sum_{i=1}^{r} \gamma_{i j}^{R} \varphi\left(\frac{c_{i j}^{R}\left(x_{j}-x_{j}^{n}\right)^{R}}{\gamma_{i j}^{R}}+\left[\mathbf{C}^{R} \mathbf{x}^{R, n}\right]_{i} ;\left[\mathbf{C}^{R} \mathbf{x}^{R, n}\right]_{i}\right), \\
& S_{j}^{I, n}\left(x_{j}^{R}\right) \\
& \quad \triangleq \beta^{I} \sum_{i=1}^{r} \gamma_{i j}^{I} \varphi\left(\frac{c_{i j}^{I}\left(x_{j}-x_{j}^{n}\right)^{I}}{\gamma_{i j}^{I}}+\left[\mathbf{C}^{I} \mathbf{x}^{I, n}\right]_{i} ;\left[\mathbf{C}^{I} \mathbf{x}^{I, n}\right]_{i}\right), \\
& S_{j}^{n}\left(x_{j}\right) \\
& \quad=S_{j}^{R, n}\left(x_{j}^{R}\right)+S_{j}^{I, n}\left(x_{j}^{I}\right),
\end{aligned}
$$

and we define $\gamma_{i j}^{o}=\left|c_{i j}^{o}\right| / \Sigma_{j^{\prime}=1}^{P}\left|c_{i j^{\prime}}^{o}\right|$, where $o$ represents $R$ or $I$. From Eq. (39), the first derivative of $\varphi(t ; s)$ evaluated at $t=s$ is $\dot{\psi}(s)$; thus the gradient of $S_{j}^{n}$ is

$$
\begin{aligned}
\nabla S_{j}^{n}\left(x_{j}^{n}\right) & =\left.\left(\begin{array}{c}
\frac{\partial}{\partial x_{j}^{R}} S_{j}^{R, n}\left(x_{j}^{R}\right) \\
\frac{\partial}{\partial x_{j}^{I}} S_{j}^{I, n}\left(x_{j}^{I}\right)
\end{array}\right)\right|_{x_{j}=x_{j}^{n}} \\
& =\left(\begin{array}{c}
\beta^{R} \sum_{i=1}^{r} c_{i j}^{R} \dot{\psi}\left(\left[\mathbf{C}^{R} \mathbf{x}^{R, n}\right]_{i}\right) \\
\beta^{I} \sum_{i=1}^{r} c_{i j}^{I} \dot{\psi}\left(\left[\mathbf{C}^{I} \mathbf{x}^{I, n}\right]_{i}\right)
\end{array}\right) \\
& =\left.\left(\begin{array}{c}
\frac{\partial}{\partial x_{j}^{R}} V(\mathbf{x}) \\
\frac{\partial}{\partial x_{j}^{I}} V(\mathbf{x})
\end{array}\right)\right|_{x=\mathbf{x}^{n}} \triangleq\left(\begin{array}{c}
\dot{V}_{j}^{R, n} \\
\dot{V}_{j}^{I, n}
\end{array}\right) .
\end{aligned}
$$

Because there is no coupling between $x_{j}^{R}$ and $x_{j}^{I}$ in the penalty or its surrogate functions, the Hessian matrix for $S_{j}^{n}$ is diagonal:

$$
\nabla^{2} S_{j}^{n}\left(x_{j}^{n}\right)=\left[\begin{array}{cc}
p_{j}^{R, n} & 0 \\
0 & p_{j}^{I, n}
\end{array}\right]
$$

where

$$
p_{j}^{o, n}=\left.\frac{\partial^{2} S_{j}^{o, n}}{\partial\left(x_{j}^{o}\right)^{2}}\right|_{x_{j}=x_{j}^{n}}=\beta^{o} \sum_{i=1}^{r} \frac{\left(c_{i j}^{o}\right)^{2}}{\gamma_{i j}^{o}} \omega\left(\left[\mathbf{C}^{o} \mathbf{x}^{o, n}\right]_{i}\right) .
$$


To obtain the update of the SPS algorithm in PL estimation, we combine the surrogates for the likelihood and the penalty. Thus the overall surrogate function to be minimized becomes

$$
\phi_{j}^{n}\left(x_{j}\right)=Q_{j}^{n}\left(x_{j}\right)+S_{j}^{n}\left(x_{j}\right) .
$$

So the update $x_{j}$ is obtained through

$$
x_{j}^{n+1}=\arg \min _{x_{j}} \phi_{j}^{n}\left(x_{j}\right) .
$$

Similar to Eq. (34), the update of the SPS algorithm with regularization becomes

$$
\begin{aligned}
& \left(\begin{array}{c}
x_{j}^{R, n+1} \\
x_{j}^{I, n+1}
\end{array}\right) \\
& =\left(\begin{array}{c}
x_{j}^{R, n} \\
x_{j}^{I, n}
\end{array}\right)-\frac{1}{\operatorname{det} \widetilde{\mathbf{H}}_{j}} \\
& \quad \times\left(\begin{array}{c}
\left(d_{j}^{I I}+p_{j}^{R, n}\right)\left(\dot{L}_{j}^{R, n}+\dot{V}_{j}^{R, n}\right)-d_{j}^{R I}\left(\dot{L}_{j}^{I, n}+\dot{V}_{j}^{I, n}\right) \\
-d_{j}^{R I}\left(\dot{L}_{j}^{R, n}+\dot{V}_{j}^{R, n}\right)+\left(d_{j}^{R R}+p_{j}^{R, n}\right)\left(\dot{L}_{j}^{I, n}+\dot{V}_{j}^{I, n}\right)
\end{array}\right),
\end{aligned}
$$

where the new Hessian matrix $\tilde{\mathbf{H}}_{j}$ is

$$
\tilde{\mathbf{H}}_{j}=\mathbf{H}_{j}+\left[\begin{array}{cc}
p_{j}^{R, n} & 0 \\
0 & p_{j}^{I, n}
\end{array}\right]
$$

and its determinant is

$$
\operatorname{det} \widetilde{\mathbf{H}}_{j}=\left(d_{j}^{R R}+p_{j}^{R, n}\right)\left(d_{j}^{I I}+p_{j}^{I, n}\right)-\left(d_{j}^{R I}\right)^{2} .
$$

The SPS algorithm outline for holographic image reconstruction is shown below (for simplicity, we suppress the " $n$ " superscript):

$$
\begin{aligned}
& \hat{\mathbf{x}}=\text { initial image } \\
& \begin{aligned}
a_{i} & =\sum_{j=1}^{P}\left|a_{i j}\right|, \quad i=1, \ldots, N \\
\text { for } n & =1, \ldots, N_{\text {iterations }} \\
& \hat{l}=\sum_{j=1}^{P} a_{i j} \hat{x}_{j}, \quad i=1, \ldots, N \\
k_{i}^{n} & =\left|\hat{l}+u_{i}\right|^{2}+b_{i}, \quad i=1, \ldots, N \\
h_{i} & =\frac{-2 y_{i}\left(\hat{l}+u_{i}\right)}{k_{i}^{n}}+2\left(\hat{l}+u_{i}\right), \quad i=1, \ldots, N .
\end{aligned}
\end{aligned}
$$

Compute $c_{i}^{R}$ and $c_{i}^{I}$ using Eq. (26)

$$
\begin{aligned}
\text { for } j & =1, \ldots, P \\
\dot{L}_{j} & =\sum_{i=1}^{N} a_{i j}^{*} \dot{h}_{i} \\
\dot{L}_{j}^{R} & =\operatorname{Re}\left\{\dot{L}_{j}\right\}, \quad \dot{L}_{j}^{I}=\operatorname{Im}\left\{\dot{L}_{j}\right\} \\
d_{j}^{R R} & =\sum_{i=1}^{N} \frac{a_{i}}{\left|a_{i j}\right|}\left[\left(a_{i j}^{R}\right)^{2} c_{i}^{R}+\left(a_{i j}^{I}\right)^{2} c_{i}^{I}\right] \\
d_{j}^{I I} & =\sum_{i=1}^{N} \frac{a_{i}}{\left|a_{i j}\right|}\left[\left(a_{i j}^{I}\right)^{2} c_{i}^{R}+\left(a_{i j}^{R}\right)^{2} c_{i}^{I}\right]
\end{aligned}
$$

$$
\begin{aligned}
& d_{j}^{R I}=d_{j}^{I R}=\sum_{i=1}^{N} \frac{a_{i} a_{i j}^{R} a_{i j}^{I}}{\left|a_{i j}\right|}\left(-c_{i}^{R}+c_{i}^{I}\right) \\
& \dot{V}_{j}^{R}=\beta^{R} \sum_{i=1}^{r} c_{i j}^{R} \dot{\psi}\left(\left[\mathbf{C}^{R} \hat{\mathbf{x}}^{R}\right]_{i}\right) \\
& \dot{V}_{j}^{I}=\beta^{I} \sum_{i=1}^{r} c_{i j}^{I} \dot{\psi}\left(\left[\mathbf{C}^{I} \hat{\mathbf{x}}^{I}\right]_{i}\right) \\
& p_{j}^{R}=\beta^{R} \sum_{i=1}^{r} \frac{\left(c_{i j}^{R}\right)^{2}}{\gamma_{i j}^{R}} \omega\left(\left[\mathbf{C}^{R} \hat{\mathbf{x}}^{R}\right]_{i}\right) \\
& p_{j}^{I}=\beta^{I} \sum_{i=1}^{r} \frac{\left(c_{i j}^{I}\right)^{2}}{\gamma_{i j}^{I}} \omega\left(\left[\mathbf{C}^{I} \hat{\mathbf{x}}^{I}\right]_{i}\right) \\
& \operatorname{det} \mathbf{H}_{j}=\left(d_{j}^{R R}+p_{j}^{R}\right)\left(d_{j}^{I I}+p_{j}^{I}\right)-\left(d_{j}^{R I}\right)^{2} \\
& \hat{x}_{j}^{R}=\hat{x}_{j}^{R}-\frac{1}{\operatorname{det} \mathbf{H}_{j}}\left[\left(d_{j}^{I I}+p_{j}^{R}\right)\left(\dot{L}_{j}^{R}+\dot{V}_{j}^{R}\right)\right. \\
& \left.\quad-d_{j}^{R I}\left(\dot{L}_{j}^{I}+\dot{V}_{j}^{I}\right)\right] \\
& \hat{x}_{j}^{I}=\hat{x}_{j}^{I}-\frac{1}{\operatorname{det} \mathbf{H}_{j}}\left[-d_{j}^{R I}\left(\dot{L}_{j}^{R}+\dot{V}_{j}^{R}\right)\right. \\
& \left.+\left(d_{j}^{I I}+p_{j}^{R}\right)\left(\dot{L}_{j}^{I}+\dot{V}_{j}^{I}\right)\right]
\end{aligned}
$$

end

end

\section{F. Number of Holograms Used}

In principle, our statistical technique can be applied to data with any number of measurement elements $N$ and to a model with any number of image pixels $P$. Whereas $N$ is fixed by the choice of the measurement device (e.g., CCD camera pixels), the value of $P$ can be selected by the algorithm designer. A natural choice for $P$ would be the number of CCD elements, which is the size of a single hologram. However, when $N<2 P$, the problem is underdetermined, so the regularization term will be particularly important. An alternative is to estimate half as many pixels as there are CCD elements, i.e., $P=N / 2$, from a single hologram. However, this option requires interpolation and downsampling processes that might introduce some artifacts into the reconstructed image. To study the effect of the sizes of the reconstructed image relative to the amount of data, we considered the following three different cases in our simulations:

Case 1. Use one hologram to reconstruct a holographic image whose size is half the number of CCD elements (half-size reconstruction), i.e., $P=N / 2$.

Case 2. Use one hologram to reconstruct a holographic image whose size is the same as the number of CCD elements (full-size reconstruction), i.e., $P=N$.

Case 3. Use two holograms to reconstruct a holographic image whose size is the same as the number of CCD elements (full-size reconstruction), i.e., $N=2 P$. 


\section{SIMULATION RESULTS}

In this section, we compare the conventional numerical reconstruction technique with our statistical reconstruction for the three cases described above. Moreover, we examined the effect of possible a priori knowledge that the object $f$ is real. We implemented this constraint by zeroing the imaginary part of $\mathbf{x}^{n}$ after each iteration.

\section{A. Effect of Numbers of Data Sets}

A $128 \times 128$ original image [Fig. 4(a)] that is complex was
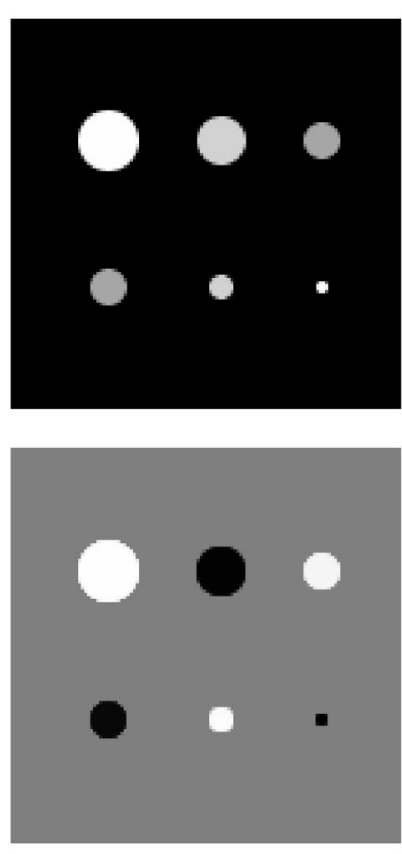

(a)
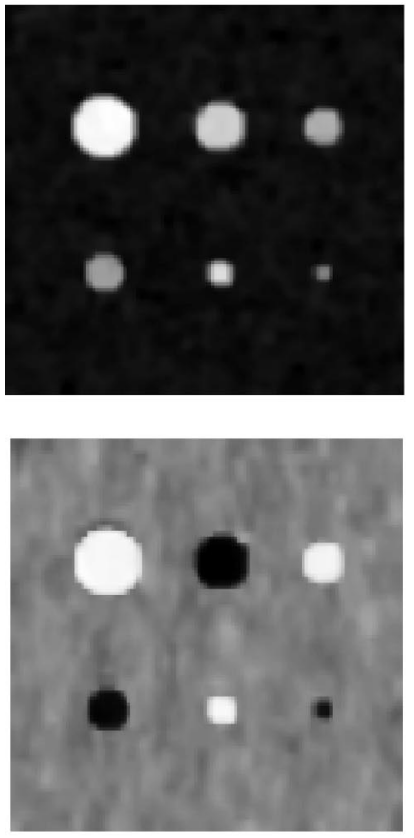

(d)
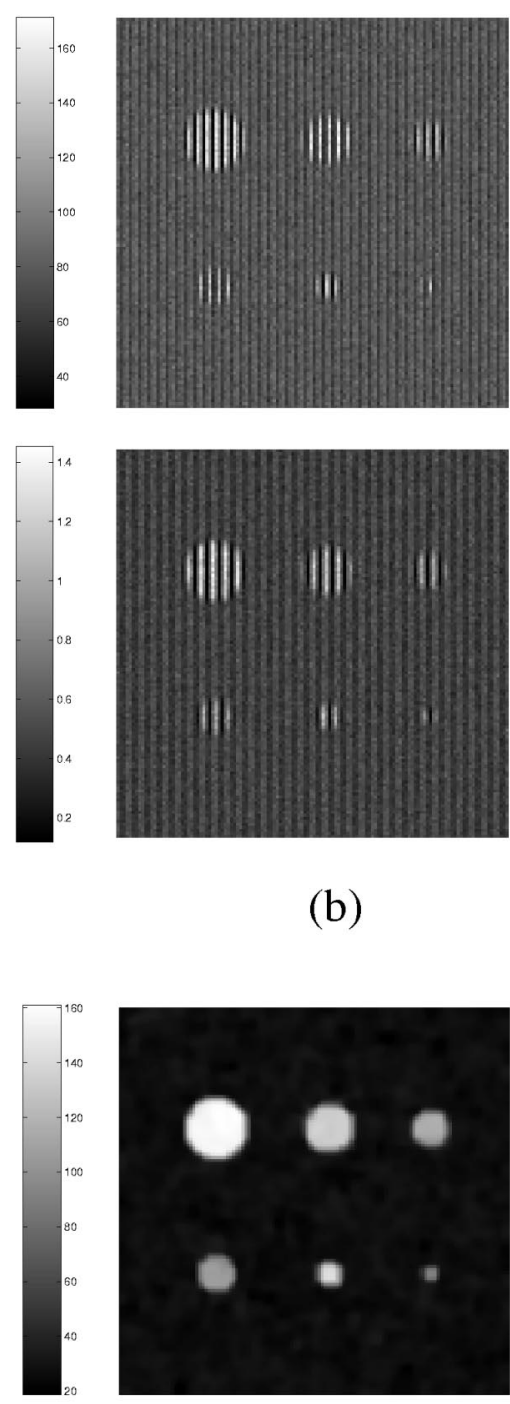

(b)
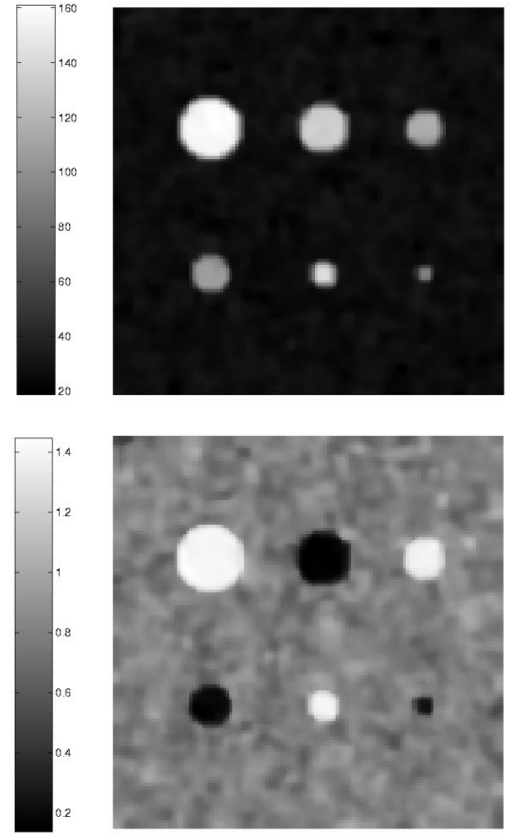

(e)
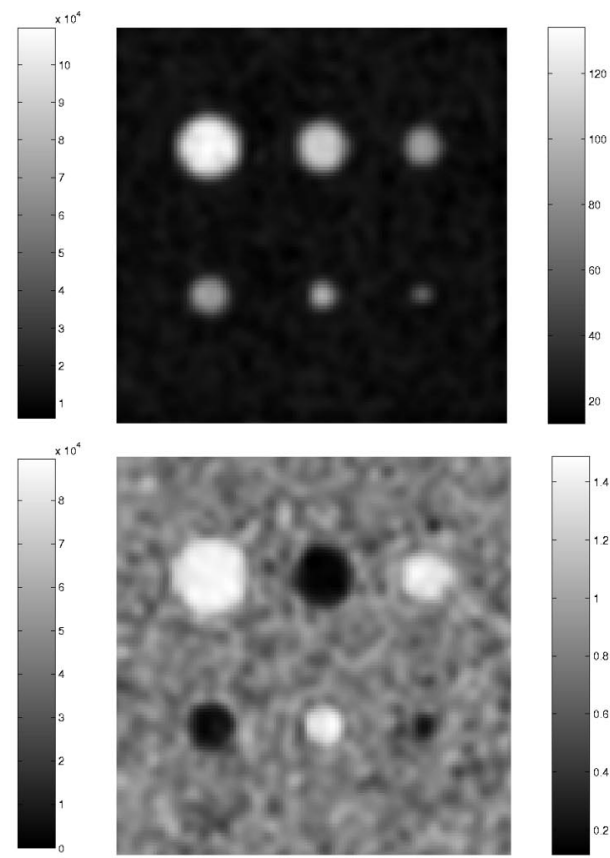

(c)
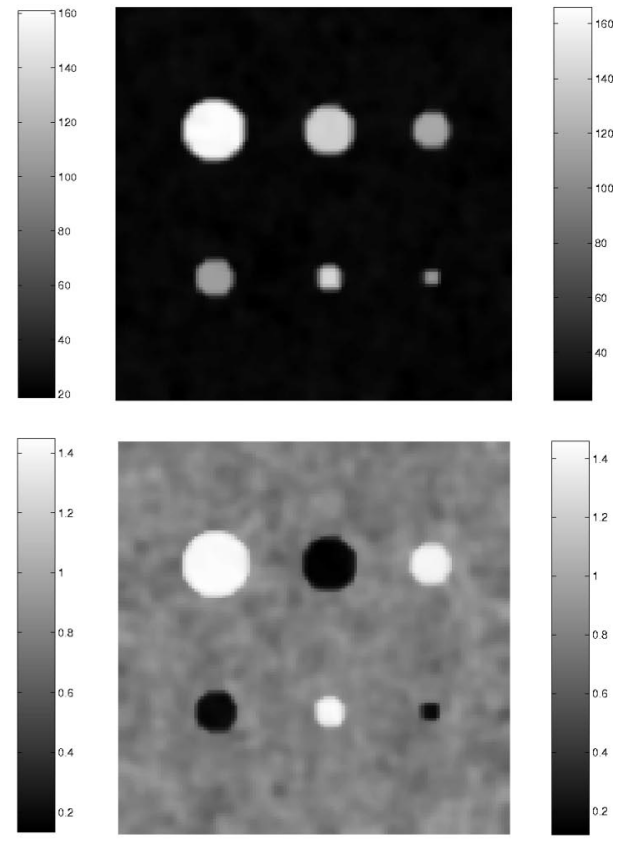

(f)

Fig. 4. Holographic reconstruction of a complex object. The top half of each pair represents the magnitude of the image, and the bottom half represents the phase of the image, except for the hologram data in (b). (a) Original image. (b) Two different holograms. (c) Conventional reconstruction using an apodizing Gaussian filter (NRMSE $=40.0 \%$ ). (d) Half-size PL reconstruction using one hologram $(\mathrm{NRMSE}=17.5 \%)$. Linear interpolation in the vertical direction to the same size as that of the original image is performed for display. (e) Full-size PL reconstruction using one hologram (NRMSE $=17.3 \%$ ). (f) Full-size PL reconstruction using two holograms $(\mathrm{NRMSE}=14.1 \%)$. 


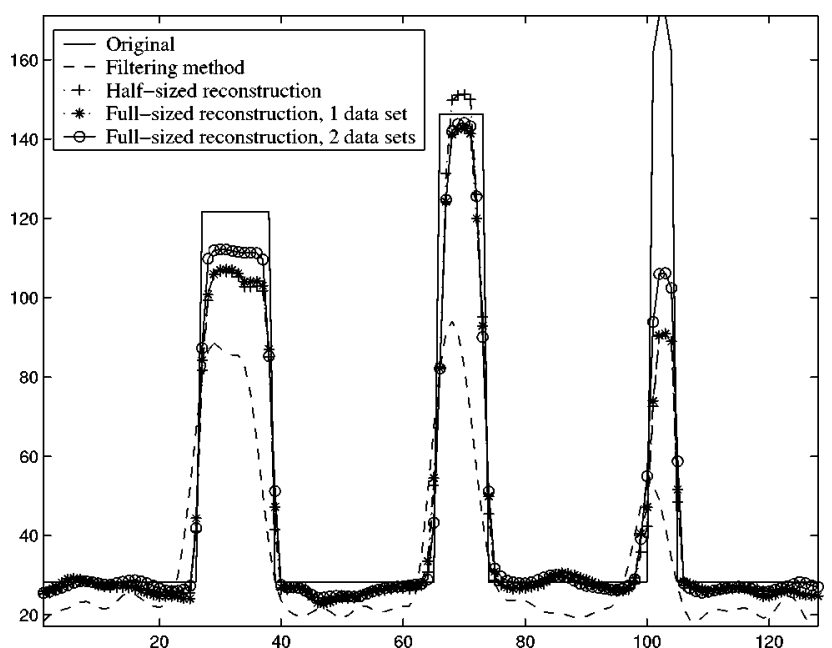

Fig. 5. Profiles of the magnitude of the numerical reconstructed images across the second row of circles.

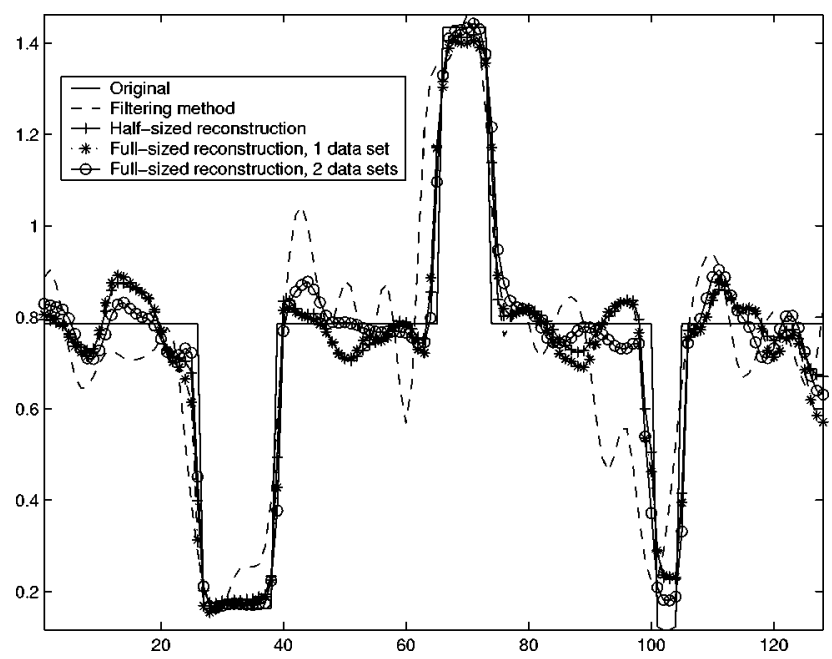

Fig. 6. Profiles of the phase of the numerical reconstructed images across the second row of circles.

degraded by the PSF, interference pattern, and Poisson noise [Fig. 4(b)], as in relation (11). We assumed a spaceinvariant optical system with a PSF that is a $7 \times 7$ jinc function, given by $J_{1}(2 \pi r) /(\pi r)$, where $J_{1}$ is a Bessel function of the first kind and $r$ is a polar-coordinate parameter, with full width at half-maximum (FWHM) of 3.5 pixels. We used the following two 2-D reference beams:

$$
\begin{aligned}
& u_{r 1}\left(n_{1}, n_{2}\right)=200 \exp \left(-i \frac{2 \pi}{3} n_{1}\right), \\
& u_{r 2}\left(n_{1}, n_{2}\right)=150 \exp \left(-i \frac{2 \pi}{4} n_{1}\right),
\end{aligned}
$$

where the pixel indices $n_{1}$ and $n_{2}$ range from 0 to 127 . For experiments with only one hologram, we used the first reference beam. The offset $b_{i}$ is assigned to be 5 and 10 for the first and the second hologram, respectively. The Poisson noise has the peak signal-to-noise ratio (PSNR) of 29 and $24 \mathrm{~dB}$ in the first and the second hologram [Fig. 4(b)], respectively. The PSNR in the data is defined as follows:

$$
\mathrm{PSNR} \triangleq 10 \log _{10}\left\{\frac{\max _{i}\left(y_{i}-b_{i}\right)^{2}}{\frac{1}{N} \sum_{i=1}^{N}\left[y_{i}-E\left(y_{i}\right)\right]^{2}}\right\} .
$$

Each simulated real-valued hologram has the same size $(128 \times 128$ pixels $)$ as that of the original complex-valued image.

Figure 4(c) shows the conventional reconstruction using an apodizing Gaussian mask. We applied a $41 \times 41$ Gaussian mask with FWHM of 27.2 pixels to the selected region in the frequency domain of the hologram. The magnitude and the phase of the reconstructed image appear to be blurry, while noise still remains. Owing to the effect of the filtering method, noise cannot be removed completely without oversmoothing edges. Figures 4(d)4(f) show our statistical holographic reconstruction based on PL estimation for the three different cases. Because of nonconvextity of the cost function $\Phi$, the reconstructed

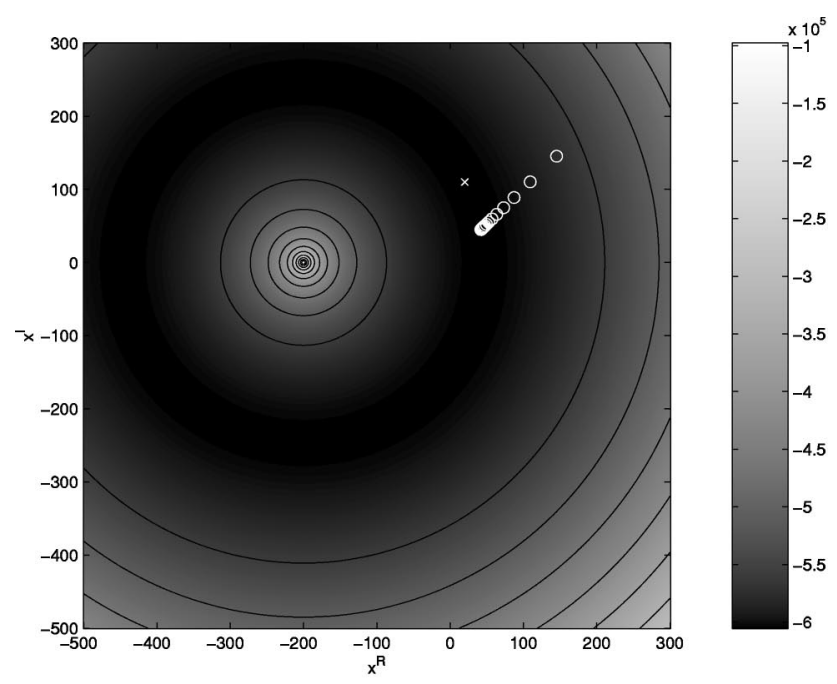

(a)

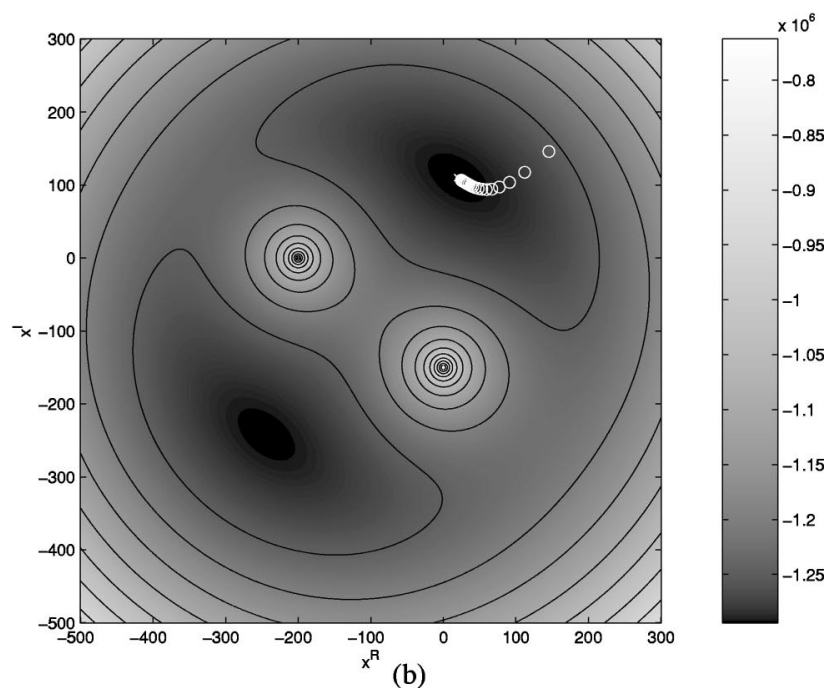

Fig. 7. Contours of the marginal objective functions at one pixel when (a) using one hologram and (b) using two holograms for full-size reconstruction. The " $\times$ " mark indicates the optimal solution at $20+i 110$, and the " $\bigcirc$ " marks indicate the updates of the estimates starting at $150+i 150$. 


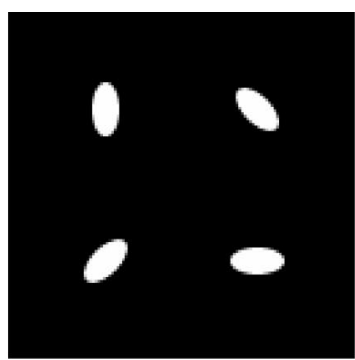

(a)

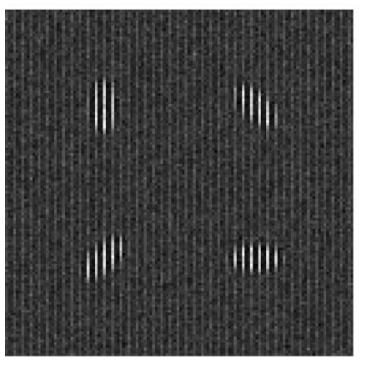

(b)

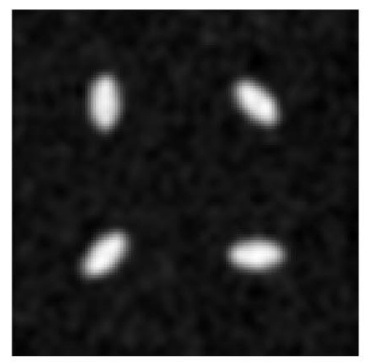

(d)

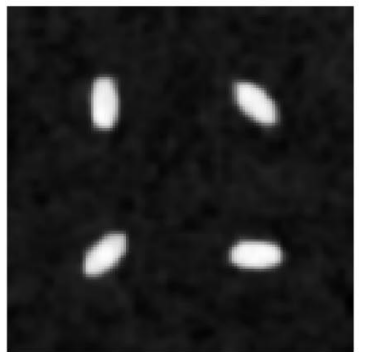

(f)

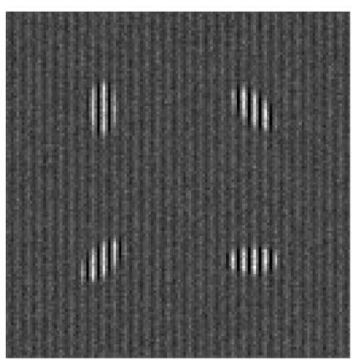

(c)

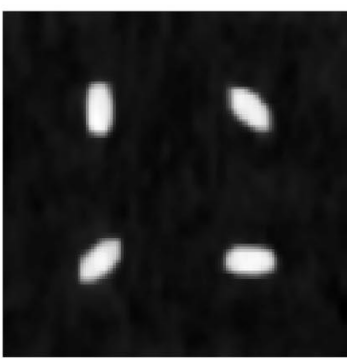

(e)

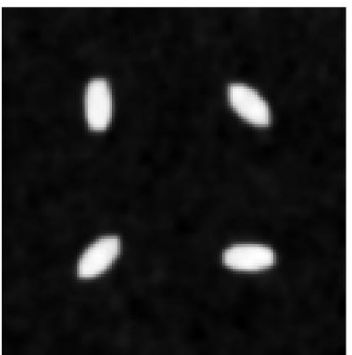

(g)
Fig. 8. PL reconstruction of a real object using the real object constraint: (a) original image, (b) and (c) hologram data, (d) conventional reconstruction using an apodizing Gaussian filter $(\mathrm{NRMSE}=43.8 \%)$, (e) half-size PL reconstruction using one hologram (NRMSE $=22.8 \%$ ), (f) full-size PL reconstruction using one hologram $(\mathrm{NRMSE}=21.1 \%)$, $(\mathrm{g})$ full-size PL reconstruction using two holograms (NRMSE $=17.2 \%)$.

image can be quite sensitive to the initial image. We used the image from the conventional approach as the initial image for the iterations. We used the nonquadratic penalty function in Eq. (16) with regularization parameters $\beta^{R}=\beta^{I}=10$ and edge-preserving parameters $\delta^{R}$ $=\delta^{I}=1$. As in most Baysian image reconstruction methods, these parameters are determined by trial and error. The SPS algorithm was run for 200 iterations, and its most expensive computation at each iteration includes five 2-D convolutions or five fast Fourier transforms. Although SPS might not be the fastest existing algorithm for this problem, the focus of the paper is to illustrate the potential of the PL reconstruction technique for digital holography. Unlike the conventional technique, the PL reconstruction technique with a nonquadratic penalty can reduce noise significantly while still preserving edges.

Figure 4(d) shows the half-size reconstruction using one hologram (case 1). For display, the reconstructed image was linearly interpolated to match the size of the original image. Figures 4(e) and 4(f) show full-size reconstructions using one and two holograms, respectively (cases 2 and 3). The half-size reconstruction has less noise but a few more artifacts than the full-size reconstruction using one hologram, perhaps as a result of interpolation and downsampling processes. Figures 5 and 6 show profiles of the magnitude and the phase of the reconstructed images. As expected, full-size reconstruction with two holograms yields the best reconstructed image with the smallest normalized root mean square error (NRMSE). The NRMSE in percent is defined as follows:

$$
\mathrm{NRMSE}=\frac{\left\|\hat{\mathbf{x}}-\mathbf{x}_{\text {true }}\right\|}{\left\|\mathbf{x}_{\text {true }}\right\|} \times 100 \%,
$$

where $\hat{\mathbf{x}}$ is the reconstructed image, $\mathbf{x}_{\text {true }}$ is the true image, and $\|\cdot\|$ denotes the Euclidean norm.

Figure 7 shows the contours of the marginal objective functions at one particular pixel for the cases of one and two holograms. For this illustration, we examined the noiseless and blurless case without regularization to clearly demonstrate how the statistical technique using two holograms can help reduce nonuniqueness. As shown in Fig. 7(a), since there are multiple minimizers, the algorithm converges to an estimate that depends strongly on the initial guess. When two holograms are used, the solutions become more distinct, and thus the algorithm often converges to the desired solution, as in Fig. 7(b). However, even with two holograms, the algorithm can converge to a limit that depends on the initial estimate because the cost function is nonconvex and may have local minima. In all cases, our statistical technique decreases the cost function monotonically, although this alone does not ensure convergence to a global minimizer for nonconvex cost functions.

\section{B. Real Object Constraint}

If the object is known a priori to be real, then we can constrain the estimate $\hat{\mathbf{x}}$ to be real by zeroing its imaginary part at each iteration. In this case, we may not need to have $N \geqslant 2 P$; since effectively the number of unknowns is reduced by a factor of 2 . Thus we expect the statistical method to yield similar results for the three cases, except possibly for some artifacts caused by interpolation for the case of half-size reconstruction. A $128 \times 128$ real image [Fig. 8(a)] was degraded by using the same parameters as those in Subsection 6.A. The conventional numerical reconstruction in Fig. 8(d) is blurry as a result of the Gaussian filter. Figures $8(\mathrm{e})-8(\mathrm{~g})$ show 200 iterations of PL reconstruction with the nonquadratic penalty function $\left(\beta^{R}\right.$ $=\beta^{I}=5, \delta^{R}=\delta^{I}=5$ ). Although the NRMSEs for all three cases differ slightly, they all provide similar reconstructed holographic images with less blur than the conventional reconstruction. Because of the real object constraint, using one hologram appears to be adequate for reconstructing a good holographic image. 


\section{CONCLUSIONS}

We have demonstrated the potential for reconstructing a digital holographic image by using the proposed statistical technique. Because the method uses all the information in the recorded hologram rather than just one term, this approach can improve the quality of the image relative to the conventional numerical reconstruction technique that uses a spatial filter applied in the spatialfrequency domain. Moreover, unlike the conventional approach, our statistical technique is not limited by the assumption of a planar reference beam. Because of the ill-conditioning and the nonuniqueness of the problem, our statistical holographic reconstruction is based on PL estimation. We constructed a statistical model for this system and developed a monotonic algorithm. Although the unique global minimum is not guaranteed because of the nonconvexity of the negative log-likelihood function in this problem, one can partially overcome the problem of multiple minima by using regularization and multiple recorded holograms. For a real object, the realness constraint can be enforced at each iteration so that the algorithm can converge faster and one hologram should be sufficient to yield a good reconstructed image.

\section{APPENDIX A}

For simplicity, we consider only the real or the imaginary part and ignore the subscript $i$ in the following proofs.

Lemma 1. If $h(l)$ and $q(l)$ are differentiable and the three conditions

$$
\begin{array}{ll}
\text { (C1) } & h(m)=q(m) \quad \text { for some } m, \\
\text { (C2) } & \dot{q}(l) \geqslant \dot{h}(l), \quad \forall l \geqslant m, \\
\text { (C3) } & \dot{q}(l) \leqslant \dot{h}(l), \quad \forall l \leqslant m
\end{array}
$$

are satisfied, then it follows that $q(l) \geqslant h(l) \forall l$ and thus $q(l)$ is a surrogate for $h(l)$, i.e., $q(l) \geqslant h(l) \forall l$.

Proof. If $l \geqslant m$, then

$$
\begin{aligned}
q(l) & =q(m)+\int_{m}^{l} \dot{q}(t) \mathrm{d} t \\
& \geqslant h(m)+\int_{m}^{l} \dot{h}(t) \mathrm{d} t=h(l) .
\end{aligned}
$$

If $l \leqslant m$, then

$$
\begin{aligned}
q(l) & =h(m)+\int_{l}^{m}[-\dot{q}(t)] \mathrm{d} t \\
& \geqslant h(m)+\int_{l}^{m}[-\dot{h}(t)] \mathrm{d} t=h(l) .
\end{aligned}
$$

Thus $q(l) \geqslant h(l) \forall l$ under the above conditions.

Lemma 2. If $h(l)$ is differentiable and the maximum

$$
c(m)=\max _{l \neq m} \frac{\dot{h}(l)-\dot{h}(m)}{l-m},
$$

is finite and nonnegative, then

$$
q(l)=h(m)+\dot{h}(m)(l-m)+\frac{1}{2} c(m)(l-m)^{2}
$$

is a parabolic surrogate for $h$, i.e., $q(l) \geqslant h(l) \forall l$.

Proof. Condition (C1) of Lemma 1 is clearly satisfied by $q$ when $l=m$. To prove condition (C2) for $l \geqslant m$, we differentiate Eq. (A3) with respect to $l$ and replace $c(m)$ with the proposed curvature as follows:

$$
\begin{aligned}
\dot{q}(l) & =\dot{h}(m)+c(m)(l-m) \\
& \geqslant \dot{h}(m)+\frac{\dot{h}(l)-\dot{h}(m)}{l-m}(l-m)=\dot{h}(l) .
\end{aligned}
$$

Similarly, $\dot{q}(l) \leqslant \dot{h}(l)$ for $l \leqslant m$, so condition (C3) is satisfied. Because all three conditions of Lemma 1 are satisfied, $q(l)$ is a parabolic surrogate for $h(l)$.

\section{APPENDIX B}

The first derivative of $h^{o}$ in Eq. (22) or (23) is

$$
\begin{aligned}
\dot{h}^{o}\left(l ; l^{n}\right)= & \frac{-2 y\left(l+u^{o}\right)\left[\left(l^{o, n}+u^{o}\right)^{2}+b / 2\right]}{k^{n}\left[\left(l+u^{o}\right)^{2}+b / 2\right]} \\
& +2\left(l+u^{o}\right) .
\end{aligned}
$$

Thus we define

$$
f(l) \triangleq \frac{\dot{h}^{o}\left(l ; l^{n}\right)-\dot{h}^{o}\left(l^{o, n} ; l^{n}\right)}{l-l^{o, n}}=\frac{2 y}{k^{n}}\left[\frac{\left(l+u^{o}\right)\left(l^{o, n}+u^{o}\right)-b / 2}{\left(l+u^{o}\right)^{2}+b / 2}\right]+2 .
$$

To obtain the maximum of the above continuous function, we equate the first derivative to zero:

$$
\dot{f}(l)=\frac{2 y}{k^{n}}\left\{\frac{-\left(l^{o, n}+u^{o}\right)\left(l+u^{o}\right)^{2}+b\left(l+u^{o}\right)+(b / 2)\left(l^{o, n}+u^{o}\right)}{\left[\left(l+u^{o}\right)^{2}+b / 2\right]^{2}}\right\}=0 .
$$

Then the optimal $l *$ that yields the maximum is

$$
l^{*}=\frac{b+\left[b^{2}+2 b\left(l^{o, n}+u^{o}\right)^{2}\right]^{1 / 2}}{2\left(l^{o, n}+u^{o}\right)}-u^{o},
$$

and

$$
f\left(l^{*}\right)=\frac{2 y\left[b^{2}+2 b\left(l^{o, n}+u^{o}\right)^{2}\right]^{1 / 2}\left(l^{o, n}+u^{o}\right)^{2}}{k^{n}\left(b^{2}+b\left\{2\left(l^{o, n}+u^{o}\right)^{2}+\left[b^{2}+2 b\left(l^{o, n}+u^{o}\right)^{2}\right]^{1 / 2}\right\}\right)}+2
$$

is the curvature of the parabolic-surrogate function. 


\section{ACKNOWLEDGMENT}

The authors thank Emmett N. Leith for helpful discussions and comments.

The authors may be reached by e-mail at the following addresses: saowapak.sotthivirat@nectec.or.th, fessler @umich.edu.

\section{REFERENCES}

1. J. W. Goodman, Introduction to Fourier Optics (McGrawHill, New York, 1996)

2. D. Gabor, "A new microscope principle," Nature (London) 161, 777-778 (1948).

3. E. N. Leith and J. Upatnieks, "Reconstructed wavefronts and communication theory," J. Opt. Soc. Am. 52, 1123-1130 (1962).

4. H. Chen, M. Shih, E. Arons, E. Leith, J. Lopez, D. Dilworth, and P. C. Sun, "Electronic holographic imaging through living human tissue," Appl. Opt. 33, 3630-3632 (1994).

5. E. Leith, H. Chen, Y. Chen, D. Dilworth, J. Lopez, R. Masri, J. Rudd, and J. Valdmanis, "Electronic holography and speckle methods for imaging through tissue using femtosecond gated pulses," Appl. Opt. 30, 4204-4210 (1991).

6. Y. Takaki and H. Ohzu, "Fast numerical reconstruction technique for high-resolution hybrid holographic microscopy," Appl. Opt. 38, 2204-2211 (1999).

7. Y. Takaki, H. Kawai, and H. Ohzu, "Hybrid holographic microscopy free of conjugate and zero-order images," Appl. Opt. 38, 4990-4996 (1999).

8. E. Cuche, F. Bevilacqua, and C. Depeursinge, "Digital holography for quantitative phase-contrasting imaging," Opt. Lett. 24, 291-293 (1999).

9. E. Cuche, P. Marquet, and C. Depeursinge, "Spatial filtering for zero-order and twin-image elimination in digital offaxis holography," Appl. Opt. 39, 4070-4075 (2000)

10. I. Yamaguchi and T. Zhang, "Phase-shifting digital holography," Opt. Lett. 22, 1268-1270 (1997).

11. S. De Nicola, P. Ferraro, A. Finizio, and G. Pierattini, "Wave front reconstruction of Fresnel off-axis holograms with compensation of aberrations by means of phase-shifting digital holography," Opt. Lasers Eng. 37, 331-340 (2002).

12. M. Liebling, T. Blu, E. Cuche, P. Marquet, C. Depeursinge, and M. Unser, "A novel non-diffractive reconstruction method for digital holographic microscopy," in Proceedings of the IEEE International Symposium on Biomedical Imaging (Institute of Electrical and Electronics Engineers, New York, 2002), pp. 625-628.

13. C. A. Bouman and K. Sauer, "A unified approach statistical tomography using coordinate descent optimization," IEEE Trans. Image Process. 5, 480-492 (1996).

14. H. Erdoğan and J. A. Fessler, "Monotonic algorithms for transmission tomography," IEEE Trans. Med. Imaging 18, 801-814 (1999).

15. J. A. Conchello, "Superresolution and convergence properties of the expectation-maximization algorithm for maximum-likelihood deconvolution of incoherent images," J. Opt. Soc. Am. A 15, 2609-2619 (1998).
16. T. J. Holmes, "Maximum-likelihood image restoration adapted for noncoherent optical imaging," J. Opt. Soc. Am. A 5, 666-673 (1988).

17. M. Cetin, W. C. Karl, and A. S. Willsky, "Edge-preserving image reconstruction for coherent imaging applications," in Proceedings of the IEEE International Conference on Image Processing (Institute of Electrical and Electronics Engineers, New York, 2002), Vol. 2, pp. 481-484.

18. B. W. Silverman, Density Estimation for Statistics and Data Analysis (Chapman \& Hall, New York, 1986).

19. J. A. Fessler and A. O. Hero, "Penalized maximumlikelihood image reconstruction using space-alternating generalized EM algorithms," IEEE Trans. Image Process. 4, 1417-1429 (1995)

20. H. L. Van Trees, Detection, Estimation, and Modulation Theory (Wiley, New York, 1968).

21. J. L. Marroquin and M. Tapia, "Parallel algorithms for phase unwrapping based on Markov random field models," J. Opt. Soc. Am. A 12, 2578-2585 (1995).

22. W. Kim and M. H. Hayes, "Phase retrieval using two Fourier-transform intensities," J. Opt. Soc. Am. A 7, 441449 (1990).

23. S. Sotthivirat and J. A. Fessler, "Image recovery using partitioned-separable paraboloidal surrogate coordinate ascent algorithms," IEEE Trans. Image Process. 11, 306-317 (2002)

24. E. Arons and E. Leith, "Coherence confocal-imaging system for enhanced depth discrimination in transmitted light," Appl. Opt. 35, 2499-2506 (1996).

25. P.-C. Sun and E. N. Leith, "Broad-source image plane holography as a confocal imaging process," Appl. Opt. 33, 597602 (1994).

26. Y. Censor, "Finite series expansion reconstruction methods," Proc. IEEE 71, 409-419 (1983).

27. D. L. Snyder, A. M. Hammoud, and R. L. White, "Image recovery from data acquired with a charge-coupled-device camera," J. Opt. Soc. Am. A 10, 1014-1023 (1993).

28. M. Cetin, W. C. Karl, and A. S. Willsky, "Edge-preserving image reconstruction for coherent imaging applications," in Proceedings of the IEEE International Conference on Image Processing (Institute of Electrical and Electronics Engineers, New York, 2002), Vol. 2, pp. 481-484.

29. M. Cetin and W. C. Karl, "Feature-enhanced synthetic aperture radar image formation based on nonquadratic regularization," IEEE Trans. Image Process. 10, 623-631 (2001).

30. K. Lange, "Convergence of EM image reconstruction algorithms with Gibbs smoothing," IEEE Trans. Med. Imaging 9, 439-446 (1990).

31. J. A. Fessler, "Grouped coordinate descent algorithms for robust edge-preserving image restoration," in Image Reconstruction and Restoration II, T. J. Schulz, ed., Proc. SPIE 3170, 184-194 (1997).

32. P. J. Huber, Robust Statistics (Wiley, New York, 1981).

33. J. A. Fessler, "Grouped-coordinate ascent algorithms for penalized-likelihood transmission image reconstruction," IEEE Trans. Med. Imaging 16, 166-175 (1997).

34. A. R. De Pierro, "A modified expectation maximization algorithm for penalized likelihood estimation in emission tomography," IEEE Trans. Med. Imaging 14, 132-137 (1995)

35. S. Sotthivirat, "Statistical image recovery techniques for optical imaging systems," Ph.D. dissertation (University of Michigan, Ann Arbor, Mich., 2003). 